\title{
Super Brønsted Acid Catalysis in Organic Synthesis
}

\author{
Hisashi Yamamoto* and Matthew B. Boxer
}

\begin{abstract}
This mini-review concerns the rapidly growing field of Brønsted acid catalysis. Brønsted acid catalysts are able to play an important role in green chemistry. Without the use of any transition metal catalysts, various organic transformations including asymmetric synthesis can be performed efficiently. This review summarizes our recent results in this field.
\end{abstract}

Keywords: Aldol · Allylation · Diels-Alder · Super Brønsted acid · Super silyl group

\section{Introduction}

Two acid-base definitions are used in organic chemistry today: The Brønsted-Lowry definition and the Lewis definition. The Brønsted-Lowry definition refers to substances that either donate protons (acid) or accept protons (base). The Lewis definition refers to electron acceptors (acid) and electron donators (base). The term 'super Brøn-

${ }^{\star}$ Correspondence: Prof. Dr H. Yamamoto

Department of Chemistry

The University of Chicago

5735 South Ellis Avenue

Chicago, IL 60637

USA

Tel.: +1 7737025059

Fax: +1 7737020805

E-Mail: yamamoto@uchicago.edu sted acid' was described by Gillespie as Brønsted acids stronger than $100 \%$ sulfuric acid. ${ }^{[1]}$ While acids of this strength can be detrimental to common reaction conditions, many of these super acids have found great use as catalysts in organic synthesis.

\section{2. $\mathrm{HNTf}_{2}$ as a Catalyst/Initiator}

One example of the high utility of these reagents has been demonstrated by the simple Mukaiyama aldol reaction as shown in Scheme 1. [2] This system was also applied to the Sakurai-Hosomi allylation of ketones and aldehydes (Scheme 2). Although the real catalyst in these reactions is not the Brønsted acid itself, but rather $\mathrm{Me}_{3} \mathrm{SiNTf}_{2}$, the high reactivity of this acid catalyst comes from the high reactivity of $\mathrm{Tf}_{2} \mathrm{NH}$ and more importantly the stability of the counter anion, $\mathrm{NTf}_{2}^{-}$.
Related to the aforementioned silyl Lewis acid catalysis, Brønsted acids and silyl Lewis acid catalysts are often inextricably linked (the Brønsted acid can be termed initiator in many cases). This comes from the facile generation of the silyl Lewis acid from super Brønsted acid and silyl enol ether (or allylsilane) through protodesilation (Scheme 3). Since $\mathrm{Me}_{3} \mathrm{SiNTf}_{2}$ is a reactive species and moisture-sensitive reagent, small amounts of water in the reaction mixture would cause decomposition to give $\mathrm{Me}_{3} \mathrm{SiOH}$ and $\mathrm{HNTf}_{2}$. The silanol, $\mathrm{Me}_{3} \mathrm{SiOH}$, that is generated will then react with $\mathrm{Me}_{3} \mathrm{SiNTf}_{2}$, and provide inert $\mathrm{Me}_{3} \mathrm{SiOSiMe}_{3}$ and HNTf2. The regenerated $\mathrm{HNTf}_{2}$ will readily react with allyltrimethylsilane (or silyl enol ether in Mukaiyama aldol reactions), and provide $\mathrm{Me}_{3} \mathrm{SiNTf}_{2}$ again. The repetition of this cycle should produce strictly anhydrous conditions. Thus this catalytic cycle constitutes a self-repairing system for Lewis acid catalysis and may

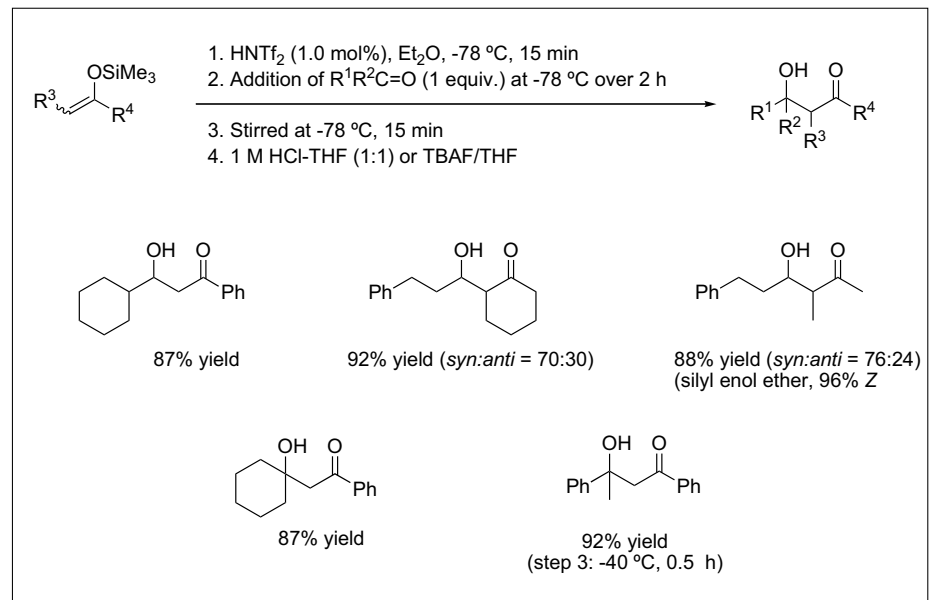

Scheme 1. $\mathrm{HNTf}_{2}$-initiated Mukaiyama aldol reaction

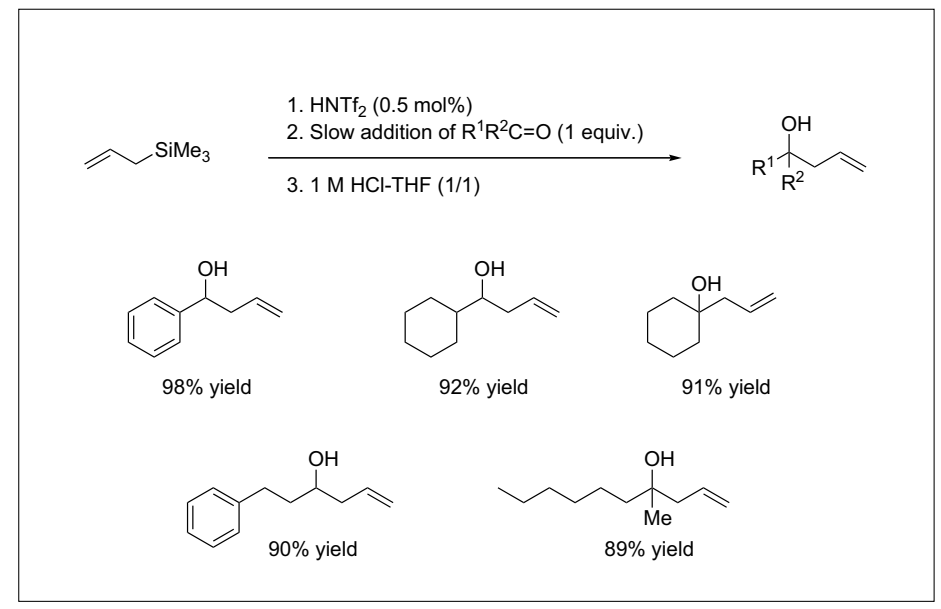

Scheme 2. Sakurai-Hosomi allylation of ketones and aldehydes 


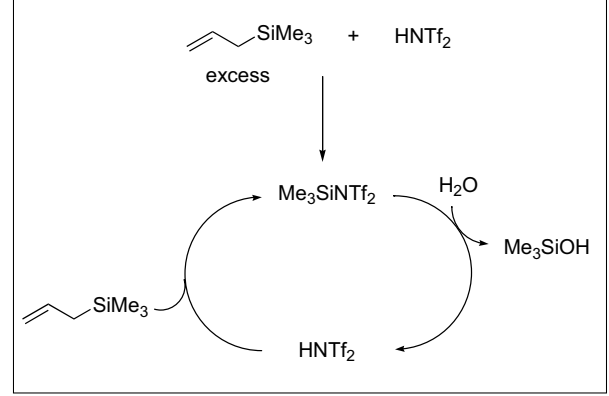

Scheme 3. Self-repair mechanism of $\mathrm{HNTf}_{2}$

help explain why particularly low catalyst loading is possible (i.e. $0.05 \mathrm{~mol} \%$ in some cases).

The trimethylsilyl (TMS) group is a widely used protecting group and Lewis acid, as well as an important functional group in many substrates. For these reasons we deemed the TMS group a first-generation silyl group. We have recently described that the use of $\mathrm{HNTf}_{2}$ as a catalyst initiator is very effective for the aldehyde crossaldol reaction. ${ }^{[3]}$ Due to the reactivity with $\mathrm{HNTf}_{2}$ that was not attainable with the classic super Brønsted acid, HOTf, we classified HNTf $_{2}$ as a 2nd generation super Brønsted acid (Scheme 4). The success of this reaction proved to be the use of triflimide as the catalyst/initiator as well as the use of tris(tri methylsilyl)silyl (TTMSS) enol ethers. The use of the TTMSS group, also referred to as the super silyl group, is one of the keys for this reaction and its unique reactivity caused us to consider it as a second-generation silyl group.

The exceptional diastereoselectivity, 'control' and reactivity of the TTMSS group can be attributed to the two classic arguments of sterics and electronics. The TTMSS group is extraordinarily bulky and has been stated to shield molecular skeletons effectively. ${ }^{[4]}$ After the first addition and silyl transfer, the steric encumberment of this group is likely to kinetically slow down the addition of a second equivalent of nucleophile to a rate that does not compete with the rate of the first addition. When all of the aldehyde starting material has been consumed, a second addition occurs giving the 2:1 (silyl enol ether:aldehyde starting material) adducts with high diastereoselectivity (Scheme 4).

Intrigued by TTMSSNTf 2 catalysis, we used ${ }^{29} \mathrm{Si} \mathrm{NMR}$ as an indicator of silicon Lewis acidity and found that the central silicon of TTMSSNTf ${ }_{2}$ was shifted significantly down-field ( $>6 \mathrm{ppm}$ ) compared to TMSand TBSNTf $_{2}$, and only slightly down-field from pentamethyldisilane-NTf $2(62.2,55.9$, 55.5, and $60.8 \mathrm{ppm}$ respectively). This trend shows a considerable difference in the cationic nature of silyl groups with only silicon-carbon bonds versus those with silicon-silicon bonds.

$$
\text { First Generation Second Generation }
$$

Scheme 4. Use of second-generation super Brønsted acid and second-generation silyl group for unprecedented aldehyde-crossaldol reaction

\section{Homogeneous and Heterogenous Carbon-based Super Brønsted Acids}

The trifluoromethanesulfonyl (triflyl, Tf) group is one of the strongest neutral electronwithdrawing groups. In particular, it greatly increases the acidity of hydrogen atoms at $\alpha$ positions. The steric and electronic factors of the aromatic ring on arylbis(triflyl)methanes are expected to greatly influence the Brønsted acidity, catalytic activity, and selectivity for organic reactions. We have developed new strong carbon Brønsted acids, pentafluoro phenylbis(triflyl)methane and polystyrenebound tetrafluorophenyl-bis(triflyl)methane (Scheme 5).[5] The synthesis of the resinbound Brønsted acid has been accomplished by using the nucleophilic para-substitution reaction of lithium pentafluorophenylbis(tri flyl)methide with lithiated polystyrenes as a key step. This is the first example of a highly acidic heterogeneous Brønsted acid catalyst that is effectively swollen by non-polar organic solvents, and its catalytic activities are superior to those of Nafion ${ }^{\circledR}$ SAC-13.

\section{Reversal of Chemoselectivity from $\mathrm{HNTf}_{2}$ to $\mathrm{B}\left(\mathrm{C}_{6} \mathrm{~F}_{5}\right)_{3}$}

In 2005 a very interesting result in the Diels-Alder reaction catalyzed by $\mathrm{HNTf}_{2}$ or Lewis acids (i.e. $\left.\mathrm{B}\left(\mathrm{C}_{6} \mathrm{~F}_{5}\right)_{3}\right)$ was found. ${ }^{[6]}$ The use of the Lewis acids provided the DielsAlder products of $\alpha, \beta$-unsaturated aldehydes and dienes selectively, while $\mathrm{HNTf}_{2}$ gave the Diels-Alder adduct of $\alpha, \beta$-unsaturated ketones and dienes preferentially (Table 1). The selectivity is believed to arise from a combination of steric as well as electronic effects of the dienophiles (Fig.).

\section{Synthesis and Application of a Chiral Brønsted Acid}

Metal-free chiral Brønsted acid catalysts have recently emerged as a new class of chiral catalysts. Several nice chiral Brønsted acids such as urea/thiourea, alcohols, and phosphoric acids have already been reported as chiral catalysts for a variety of

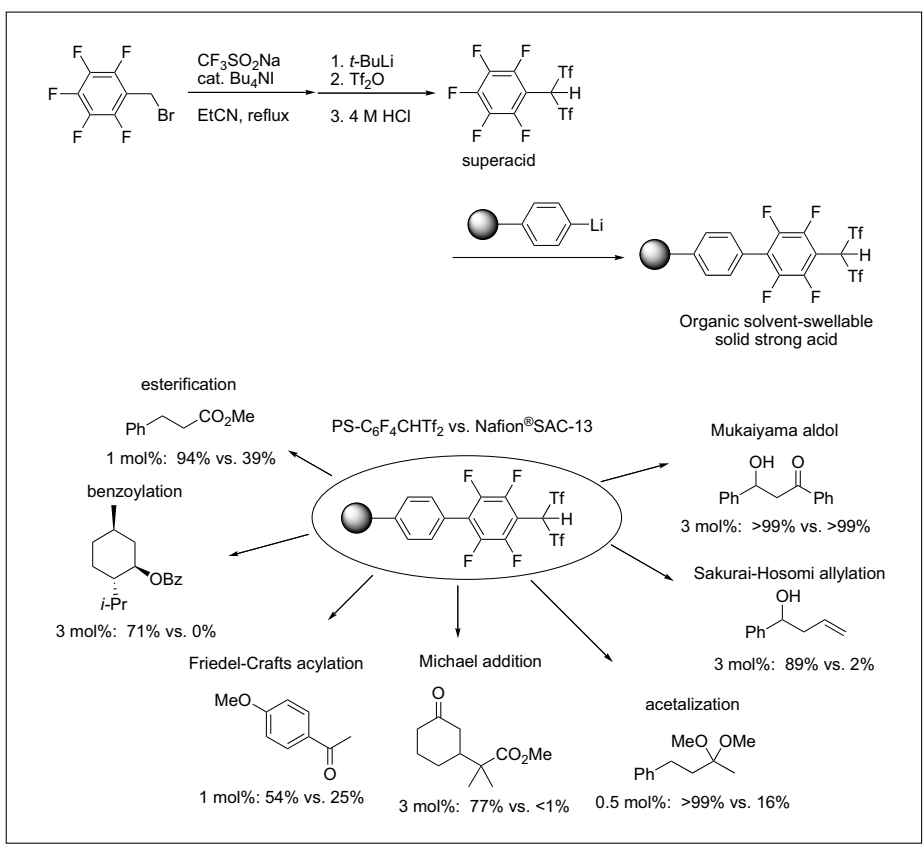

Scheme 5. Synthesis and utility of polystyrene-bound tetrafluorophenylbis(triflyl)methane 
Table 1. Reversal of chemoselectivty between $\mathrm{HNTf}_{2}$ and $\mathrm{B}\left(\mathrm{C}_{6} \mathrm{~F}_{5}\right)_{3}$

\begin{tabular}{|c|c|c|c|c|}
\hline$\pi^{\mathrm{CHO}}$ & Et & $\begin{array}{c}\begin{array}{c}\text { Catalyst } \\
\text { Diene (1 equiv.) }\end{array} \\
\mathrm{CH}_{2} \mathrm{Cl}_{2}\end{array}$ & $\begin{array}{c}\text { Diels-Alder } \\
\text { Adduct } \\
\text { of Acrolein } \\
\text { A }\end{array}$ & $\begin{array}{c}\text { Diels-Alder } \\
\text { Adduct } \\
\text { of Ethyl Vinyl Ketone } \\
\text { B }\end{array}$ \\
\hline entry & Diene & Conditions & Catalyst [mol\%] & Yield [\%], Ratio of A:B \\
\hline 1 & & $-78^{\circ} \mathrm{C}, 1 \mathrm{~h}$ & $\mathrm{Tf}_{2} \mathrm{NH}(5)$ & $86,12: 88$ \\
\hline 2 & & $-96^{\circ} \mathrm{C}, 1 \mathrm{~h}$ & $\mathrm{Tf}_{2} \mathrm{NH}(5)$ & $93,5: 95$ \\
\hline 3 & & $-78^{\circ} \mathrm{C}, 1 \mathrm{~h}$ & $\mathrm{~B}\left(\mathrm{C}_{6} \mathrm{~F}_{5}\right)_{3}(50)$ & $88,85: 15$ \\
\hline 4 & & $-40^{\circ} \mathrm{C}, 2 \mathrm{~h}$ & $\mathrm{Tf}_{2} \mathrm{NH}(5)$ & $64,39: 61$ \\
\hline 5 & & $-40^{\circ} \mathrm{C}, 2 \mathrm{~h}$ & $\mathrm{~B}\left(\mathrm{C}_{6} \mathrm{~F}_{5}\right)_{3}(30)$ & $85,94: 6$ \\
\hline 6 & & $-40^{\circ} \mathrm{C}, 2 \mathrm{~h}$ & $\mathrm{Tf}_{2} \mathrm{NH}(5)$ & $81,19: 81$ \\
\hline 7 & & $-40^{\circ} \mathrm{C}, 2 \mathrm{~h}$ & $\mathrm{~B}\left(\mathrm{C}_{6} \mathrm{~F}_{5}\right)_{3}(30)$ & $75,95: 5$ \\
\hline 8 & & $-40^{\circ} \mathrm{C}, 4 \mathrm{~h}$ & $\mathrm{Tf}_{2} \mathrm{NH}(5)$ & $51,22: 78$ \\
\hline 9 & & $-40^{\circ} \mathrm{C}, 4 \mathrm{~h}$ & $\mathrm{~B}\left(\mathrm{C}_{6} \mathrm{~F}_{5}\right)_{3}(30)$ & $62,91: 10$ \\
\hline
\end{tabular}

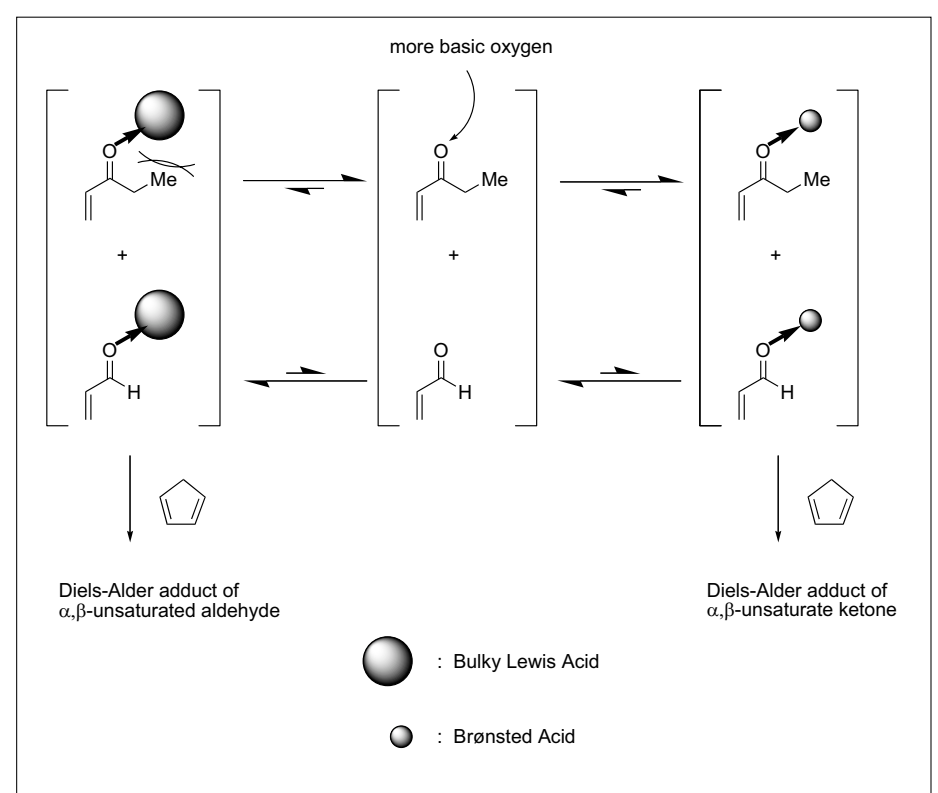

Fig. Rationale for reversal of chemoselectivity based on size of acid catalyst carbonyl and imine compounds. However, compared with chiral metal Lewis acid catalysts, the utility of these chiral Brønsted acid catalysts is still limited to reactive substrates. This drawback can be overcome by designing the Brønsted acid to have higher acidity, which in turn is one of the most important yet challenging tasks for Brønsted acid chemistry. In order to increase the acidity of Brønsted acids, it is necessary to increase the stability of the counter anion. We expected that strong chiral Brønsted acids could be obtained by introduction of $\mathrm{a}=$ NTf group into a phosphoric acid moiety. We succeeded in preparing this chiral super Brønsted acid catalyst which is able to catalyze the asymmetric Diels-Alder reaction with $\alpha, \beta$-unsaturated ketones (Table 2). ${ }^{[7]}$ This highly acidic Brønsted acid is capable of catalyzing the title reaction with only $5 \mathrm{~mol} \%$ loading, which is a relatively low catalyst loading for a chiral organic Brønsted acid.

\section{Conclusions and Outlook}

The usefulness of the triflyl (Tf) group, specifically in $-\mathrm{NTf}_{2}$ and $\mathrm{RHC}\left(\mathrm{Tf}_{2}\right)_{2}$, has been demonstrated in this review. Many of the compounds containing these groups can be considered super Brønsted acids. These super Brønsted acids are capable of catalyzing important organic transformations previously only attainable with sometimes expensive and toxic metals. The application of these types of acids in a catalytic asymmetric platform has also been studied and progress towards a widely applicable chiral Brønsted acid catalyst is still being developed.
Table 2. Diels-Alder reaction with chiral Brønsted acid
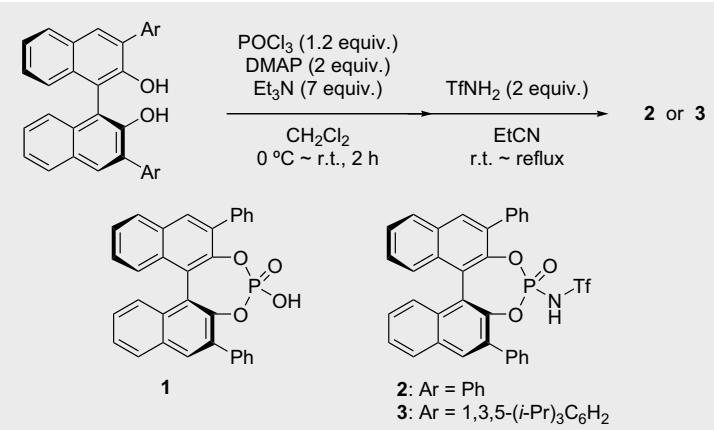

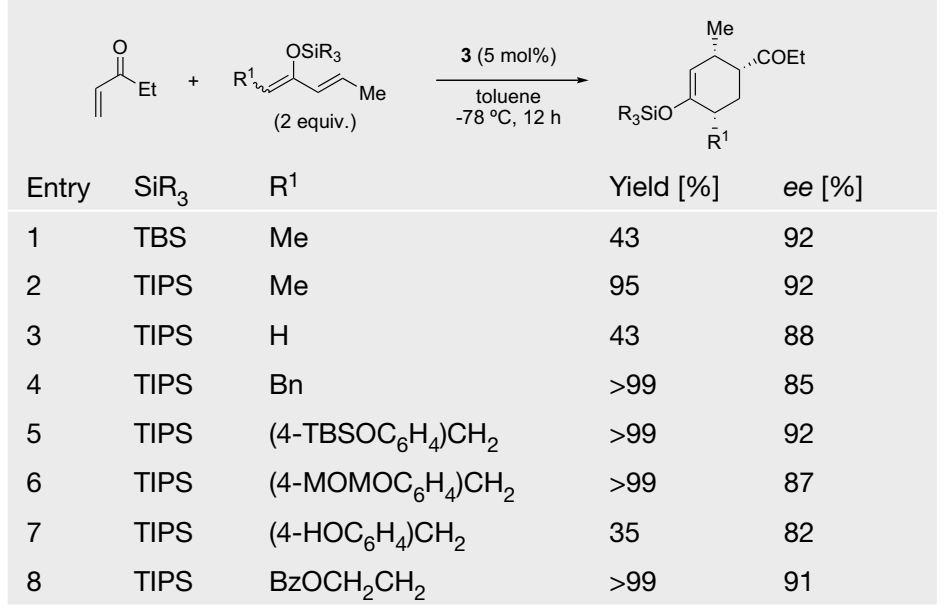

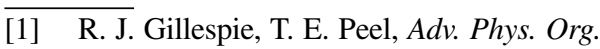
Chem. 1972, 9, 1.

[2] K. Ishihara, Y. Hiraiwa, H. Yamamoto, Synlett 2001, 1851.

[3] a) M. B. Boxer, H. Yamamoto, J. Am. Chem. Soc. 2006, 128, 48; b) M. B. Boxer, H. Yamamoto, J. Am. Chem. Soc. 2007, in press.

[4] a) H. Bock, J. Meuret, R. Baur, K. Ruppert, J. Organometal. Chem. 1993, 446, 113; b) J. Frey, E. Schottland, Z. Rappoport, D. Bravo-Zhivotovskii, M. Nakash, M. Botoshansky, M. Kaftory, Y. Apeloig, J. Chem. Soc., Perkin Trans. 2 1994, 2555.
[5] a) K. Ishihara, A. Hasegawa, H. Yamamoto, Angew. Chem., Int. Ed. 2001, 40, 4077; b) K. Ishihara, A. Hasegawa, H. Yamamoto, Synlett 2002, 1296; c) K. Ishihara, A. Hasegawa, H. Yamamoto, Synlett 2002, 1299; d) A. Hasegawa, T. Ishikawa, K. Ishihara, H. Yamamoto, Bull. Chem. Soc. Jpn. 2005, 78, 1401.

[6] D. Nakashima, H. Yamamoto, Org. Lett. 2005, 7, 1251.

[7] D. Nakashima, H. Yamamoto, J. Am. Chem. Soc. 2006, 128, 9626 